\title{
Closed chains of conics carrying poncelet triangles
}

\author{
Lorenz Halbeisen ${ }^{1}$ • Norbert Hungerbühler ${ }^{1}$
}

Received: 14 November 2016 / Accepted: 12 December 2016 / Published online: 18 January 2017 (C) The Author(s) 2017. This article is published with open access at Springerlink.com

\begin{abstract}
We investigate closed chains of conics which carry Poncelet triangles. In particular, we show that every chain of conics which carries Poncelet triangles can be closed. Furthermore, for $k=3$ and $k=4$ we show that there are closed chains of pairwise conjugate conics which carry Poncelet $k$-gons such that the contact points of each $k$-gon are the vertices of the next $k$-gon-such miraculous chains of conics do not exist for $5 \leq k \leq 23$.
\end{abstract}

Keywords Conic sections · Poncelet theorem · Conjugate conics · Projective maps

Mathematics Subject Classification 51A05 - 51A10 $\cdot$ 51A20

\section{Introduction}

In 1813, while Poncelet was in captivity as war prisoner in the Russian city of Saratov, he discovered his famous closing theorem which, in its simplest form, reads as follows (see Poncelet 1822): Let $K$ and $C$ be two conics in general position. Suppose there is a $k$-sided polygon inscribed in $K$ and circumscribed about $C$. Then for any point $P$ on $K$ which is an exterior point of $C$, there exists a (possibly degenerate) $k$-sided polygon, also inscribed in $K$ and circumscribed about $C$, which has $P$ as one of its vertices. See for example Dragović and Radnović (2011) or Flatto (2009) for classical

\footnotetext{
$\triangle$ Norbert Hungerbühler

norbert.hungerbuehler@math.ethz.ch

Lorenz Halbeisen

lorenz.halbeisen@math.ethz.ch

1 Department of Mathematics, ETH Zentrum, Rämistrasse 101, 8092 Zurich, Switzerland
} 
overviews about Poncelet's Theorem, or Halbeisen and Hungerbühler (2015) for a new elementary proof based only on Pascal's Theorem.

Recent years have seen a flourishing revitalization of Poncelet's closure theorem. We just mention some of these developments: Bos et al. (1987) gave a comprehensive overview reaching from the pre-history of Poncelet's Porism to the modern approaches using elliptic curves. In their paper they use the language of algebraic geometry to analyze steps in the proofs of Poncelet and Jacobi with the help of modern notation and methods. Del Centina (2016a, b) comments on the full range of the historical genesis of Poncelet's Theorem, its proofs, variants and relatives, and puts the results in a modern context. He does not miss to mention lesser known aspects like Nicola Trudi's approach or George Halphen's and Francesco Gerbaldi's discovery of a relation to continued fractions, or the applications of Poncelet's closure theorem and its generalizations to the theory of integrable systems, billiard dynamics, PDEs and statistical mechanics. In the further development of Poncelet's Porism, even very classical results came to new life: Chapple (1749) found a relation for the radii and the distance of the centers of two nested circles which carry Poncelet triangles. This was generalized to Poncelet $n$-gons without self-intersections for small values of $n$ by Euler (1767), Fuss (1795) and Steiner (1827a, p. 96; 1827b, p. 289). These results have recently been extended by Cieślak et al. (2013) who formulated a relation for 5-gons with self-intersections and found a link to the rotation index of bar billiards and special functions leading to a new series expansion of $\pi$. Another approach to Poncelet's Porism uses measure theory: King (1994) observed that there exists a measure on the conic which carries the vertices, which is invariant under tangential projection with respect to the inscribed conic. This easily proves Poncelet's Theorem. A similar construction has been proposed by Bryant (2010), and Cieślak and Mozgawa (2016) found a nice geometric interpretation of the corresponding measure on the inscribed conic. Vallès (2012) devised a new proof of Poncelet's Porism and of the related Darboux Theorem based upon vector bundles by exploiting a link between Schwarzenberger bundles and Poncelet curves.

In the present paper we investigate chains of conics $G_{0}, \ldots, G_{n-1}$ such that for each $0 \leq i \leq n-2$ there is a Poncelet triangle $\Delta_{i}$ with vertices on $G_{i}$ whose sides are tangent to $G_{i+1}$. It will be shown in Sect. 4 that each such chain can be closed by adding a suitable conic $G_{n}$, i.e., there is a Poncelet triangle $\Delta_{n-1}$ with vertices on $G_{n-1}$ and sides tangent to $G_{n}$, and $G_{n}$ carries the vertices of a Poncelet triangle $\Delta_{n}$ whose sides are tangent to $G_{0}$. The crucial point in the proof will be, that in specific cases, two conics can be projectively mapped simultaneously into two circles. This technical point is of some interest in its own right and will be investigated in Sect. 3.

We then show in Sect. 5 that there are closed chains of arbitrary length of conics $G_{0}, \ldots, G_{n-1}$ carrying Poncelet triangles, such that the contact points of the Poncelet triangle $\Delta_{i}$ (having its vertices on $G_{i}$ ) are the vertices of the Poncelet triangle $\Delta_{i+1}$ (having its vertices on $G_{i+1}$, where indices are taken modulo $n$ ).

Finally, in Sect. 6 we investigate two very peculiar chains of conics carrying Poncelet triangles or Poncelet quadrilaterals, respectively, which move synchronously while keeping contact with the neighbouring polygons. We also show that, up to projective transformations, these two chains are unique. Moreover, we show that there are no such chains of conics carrying $k$-gons for $5 \leq k \leq 23$. 


\section{Preliminary}

In order to make this text self-contained, we start with a brief description of the general setting and establish the notation. An extensive survey about algebraic representations of conics in the real projective plane can be found in Bix (2006) or Lord (2012).

\subsection{Projective plane and conics}

In this paper, we mostly work in the standard model of the real projective plane. For this, we consider $\mathbb{R}^{3}$ and its dual space $\left(\mathbb{R}^{3}\right)^{*}$ of linear functionals on $\mathbb{R}^{3}$. The set of points is $\mathbb{P}=\mathbb{R}^{3} \backslash\{0\} / \sim$, where $x \sim y \in \mathbb{R}^{3} \backslash\{0\}$ are equivalent, if $x=\lambda y$ for some $\lambda \in \mathbb{R}$. The set of lines is $\mathbb{B}=\left(\mathbb{R}^{3}\right)^{*} \backslash\{0\} / \sim$, where $g \sim h \in\left(\mathbb{R}^{3}\right)^{*} \backslash\{0\}$ are equivalent, if $g=\lambda h$ for some $\lambda \in \mathbb{R}$. Finally, we say a point $[x]$ and a line $[g]$ are incident if $g(x)=0$, where we denoted equivalence classes by square brackets. In the sequel we will identify $\mathbb{R}^{3}$ and $\left(\mathbb{R}^{3}\right)^{*}$ by the standard inner product $\langle\cdot, \cdot\rangle$ which allows to express the incidence through the relation $\langle x, g\rangle=0$.

As usual, a line $[g]$ can be identified with the set of points which are incident with it. Vice versa a point $[x]$ can be identified with the set of lines which pass through it. The affine plane $\mathbb{R}^{2}$ is embedded in the present model of the projective plane by the map

$$
\left(\begin{array}{l}
x_{1} \\
x_{2}
\end{array}\right) \mapsto\left[\left(\begin{array}{c}
x_{1} \\
x_{2} \\
1
\end{array}\right)\right]
$$

The projective general linear group $\operatorname{PGL}(3, \mathbb{R})$ consists of equivalence classes $[A]$ of regular matrices $A \in \mathbb{R}^{3 \times 3}$ representing maps $\mathbb{P} \rightarrow \mathbb{P},[x] \mapsto[A x]$, where two matrices are equivalent, $A_{1} \sim A_{2}$, if $A_{1}=\lambda A_{2}$ for some $\lambda \in \mathbb{R}$.

A conic in the constructed model is an equivalence class of a regular, linear, selfadjoint map $A: \mathbb{R}^{3} \rightarrow \mathbb{R}^{3}$ with mixed signature, i.e., $A$ has eigenvalues of both signs. It is convenient to say, a matrix $A$ is a conic, instead of $A$ is a representative of a conic. We may identify a conic by the set of points $[x]$ such that $\langle x, A x\rangle=0$, or by the set of lines $[g]$ for which $\left\langle A^{-1} g, g\right\rangle=0$ (see below). Notice that, in this interpretation, a conic cannot be empty: Since $A$ has positive and negative eigenvalues, there are points $[p],[q]$ with $\langle p, A p\rangle>0$ and $\langle q, A q\rangle<0$. Hence a continuity argument guarantees the existence of points $[x]$ satisfying $\langle x, A x\rangle=0$.

From now on, we will only distinguish in the notation between an equivalence class and a representative if necessary.

Fact 2.1 Let $x$ be a point on the conic $A$. Then the line $A x$ is tangent to the conic $A$ with contact point $x$.

Proof We show that the line $A x$ meets the conic $A$ only in $x$. Suppose otherwise, that $y \nsim x$ is a point on the conic, i.e., $\langle y, A y\rangle=0$, and at the same time on the line $A x$, i.e., $\langle y, A x\rangle=0$. By assumption, we have $\langle x, A x\rangle=0$. Note, that $A x \nsim A y$ since $A$ is regular, and $\langle A y, x\rangle=0$ since $A$ is selfadjoint. Hence $x$ and $y$ both are perpendicular to the plane spanned by $A x$ and $A y$, which contradicts $y \nsim x$. 
In other words, the set of tangents of a conic $A$ is the image of the points on the conic under the map $A$. And consequently, a line $g$ is a tangent of the conic iff $A^{-1} g$ is a point on the conic, i.e., if and only if $\left\langle A^{-1} g, g\right\rangle=0$.

Definition 2.2 If $P$ is a point, the line $A P$ is called its polar with respect to a conic $A$. If $g$ is a line, the point $A^{-1} g$ is called its pole with respect to the conic $A$.

Obviously, the pole of the polar of a point $P$ is again $P$, and the polar of the pole of a line $g$ is again $g$. Moreover:

Fact 2.3 If the polar of point $P$ with respect to a conic $A$ intersects the conic in a point $x$, then the tangent in $x$ passes through $P$.

Proof For $x$, we have $\langle x, A x\rangle=0$ since $x$ is a point on the conic, and $\langle x, A P\rangle=0$ since $x$ is a point on the polar of $P$. The tangent in $x$ is the line $A x$, and indeed, $P$ lies on this line, since $\langle P, A x\rangle=\langle A P, x\rangle=0$.

\subsection{Coordinate transformations and projective maps}

An element $T \in P G L(3, \mathbb{R})$ can be interpreted as a change of coordinates $x=$ $T y$ : If for example $\langle x, A x\rangle=0$ is a conic in $x$-coordinates, then $0=\langle x, A x\rangle=$ $\langle T y, A T y\rangle=\left\langle y, T^{\top} A T y\right\rangle$ is the same conic in $y$-coordinates (i.e., the transformed conic is represented by the matrix $\left.T^{\top} A T\right)$. Similarly, if $\langle g, x\rangle=0$ is a line, then $0=\langle g, x\rangle=\left\langle T^{\top} g, y\right\rangle$, i.e., the transformed line is represented by $T^{\top} g$.

Instead of considering $T$ as a coordinate transformation, we can equivalently interpret $T: \mathbb{P} \rightarrow \mathbb{P}$ as a projective map: Then, if $y$ is a point on the conic $T^{\top} A T$, i.e., if $\left\langle y, T^{\top} A T y\right\rangle=0$, then the image $x:=T y$ is a point of the conic $\langle x, A x\rangle=0$.

\subsection{Rank one and rank two matrices}

For completeness we recall the following basic facts:

Lemma 2.4 Let $A \in \mathbb{R}^{n \times n}$ be symmetric. Then:

- $A$ is of rank 1 iff $A=\alpha a a^{\top}$ for a unit vector $a \in \mathbb{R}^{n}$ and some $\alpha \in \mathbb{R} \backslash\{0\}$. Moreover, if $A=\alpha a a^{\top}$ then, the vector $a$ is an eigenvector and $\alpha$ the corresponding eigenvalue of $A$, and $\langle x, A x\rangle=\alpha\langle a, x\rangle^{2}$.

- $A$ is of rank 2 iff $A=\alpha a a^{\top}+\beta b b^{\top}$ for two orthonormal vectors $a, b \in \mathbb{R}^{n}$ and some $\alpha, \beta \in \mathbb{R} \backslash\{0\}$. Moreover, if $A=\alpha a a^{\top}+\beta b b^{\top}$ then, $a$ and $b$ are eigenvectors and $\alpha, \beta$ the corresponding eigenvalues of A. Moreover, if $A$ is of rank 2, $A=u v^{\top}+v u^{\top}$ for two vectors $u, v \in \mathbb{R}^{n} \backslash\{0\}$ iff A has mixed signature. Finally, if $A=\alpha a a^{\top}+\beta b b^{\top}$, then $\langle x, A x\rangle=\alpha\langle x, a\rangle^{2}+\beta\langle x, b\rangle^{2}$, and if $A=u v^{\top}+v u^{\top}$, then $\langle x, A x\rangle=2\langle x, u\rangle\langle x, v\rangle$.

Proof Since $A$ is symmetric there exists an orthonormal eigenbasis $t^{(1)}, \ldots, t^{(n)}$, i.e., if $T$ is the matrix with columns $t^{(1)}, \ldots, t^{(n)}$, then $T^{\top} A T=\operatorname{diag}\left(\lambda_{1}, \ldots, \lambda_{n}\right)=: D$, where $\lambda_{i}$ is the eigenvalue corresponding to $t^{(i)}$. Therefore $A=T D T^{\top}$. If $A$ is of 
rank 1, exactly one eigenvalue $\lambda_{i} \neq 0$, and hence $A=\lambda_{i} t^{(i)} t^{(i) \top}$. If $A$ is of rank 2, exactly two eigenvalues $\lambda_{i}, \lambda_{j} \neq 0$, and hence $A=\lambda_{i} t^{(i)} t^{(i) \top}+\lambda_{j} t^{(j)} t^{(j) \top}$.

On the other hand, if $A=\alpha a a^{\top}$ for a unit vector $a$ and $\alpha \in \mathbb{R}^{n} \backslash\{0\}$, it is clear that $A=A^{\top}$, that all columns of $A$ are multiples of $a$ and hence that $A$ is of rank 1. Moreover, $A a=\alpha a a^{\top} a=\alpha a$. If $A=\alpha a a^{\top}+\beta b b^{\top}$ for orthonormal vectors $a, b$ and $\alpha, \beta \in \mathbb{R} \backslash\{0\}$, then $A=A^{\top}, A a=\left(\alpha a a^{\top}+\beta b b^{\top}\right) a=\alpha a$ and $A b=$ $\left(\alpha a a^{\top}+\beta b b^{\top}\right) b=\beta b$. Hence, $A$ has two orthonormal eigenvectors and therefore rank $A \geq 2$. On the other hand, $A x=0$ whenever $x \perp \operatorname{span}(a, b)$, i.e., $\operatorname{rank} A=2$.

Finally, let $A$ be of rank 2, i.e., $A=\alpha a a^{\top}+\beta b b^{\top}$, and suppose $\alpha>0>\beta$. Then, for $u=\mu a+v b, v=\mu a-v b$ we have $A=u v^{\top}+v u^{\top}$ if we choose $\mu=\sqrt{\alpha / 2}, v=\sqrt{-\beta / 2}$. On the other hand, if $A=u v^{\top}+v u^{\top}$ for two non-zero vectors $u \neq v \in \mathbb{R}^{n}$, then $A$ has the eigenvalues

$$
0,\langle u, v\rangle+\|u\|\|v\|,\langle u, v\rangle-\|u\|\|v\| .
$$

Thus, by the Cauchy-Schwarz inequality, the two non-zero eigenvalues have opposite sign.

The formulas for the quadratic forms follow immediately.

Lemma 2.5 Let $A$ be a conic and $C \in \mathbb{R}^{3 \times 3}$ symmetric of rank 1. Suppose, $A+C$ is also a conic, and $p$ a point on $A$ and on $A+C$. Then, $A$ and $A+C$ have a common tangent in $p$.

Proof According to Lemma 2.4, $C$ is of the form $\gamma c c^{\top}$ for some $c \in \mathbb{R}^{3} \backslash\{0\}, \gamma \neq 0$. We have

$$
0=\langle p,(A+C) p\rangle=\langle p, A p\rangle+\langle p, C p\rangle=\langle p, C p\rangle=\gamma\langle c, p\rangle^{2},
$$

hence $\langle c, p\rangle=0$. The tangent in $p$ at $A$ is given by $0=\langle x, A p\rangle$. The tangent in $p$ at $A+C$ is given by the same equation: $0=\langle x,(A+C) p\rangle=\left\langle x, A p+\gamma c c^{\top} p\right\rangle=$ $\langle x, A p\rangle$.

\subsection{Projective maps leaving a circle invariant}

Let $K=\operatorname{diag}(1,1,-1)$ be the affine unit circle and $\mathfrak{G}_{K}$ the subgroup of all projective maps which leave $K$ invariant, i.e.,

$$
\mathfrak{G}_{K}:=\left\{T \in P G L(3, \mathbb{R}) \mid T^{\top} K T \sim K\right\}
$$

Lemma 2.6 If $T \in \mathfrak{G}_{K}$ has the fixed points $(0,0,1)^{\top}$ and $(1,0,1)^{\top}$, then $T$ is the identity or $T=\operatorname{diag}(1,-1,1)=: S$.

Proof Since $(0,0,1)^{\top}$ is a fixed point of $T$, we have

$$
T=\left(\begin{array}{ccc}
* & * & 0 \\
* & * & 0 \\
t_{31} & * & t_{33}
\end{array}\right)
$$


Then, because $(1,0,1)^{\top}$ is also a fixed point, if follows

$$
T=\left(\begin{array}{ccc}
t_{31}+t_{33} & * & 0 \\
0 & * & 0 \\
t_{31} & * & t_{33}
\end{array}\right)
$$

i.e., $t_{11}=t_{31}+t_{33}$. On the other hand, from $K \sim T^{\top} K T$, it now follows

$$
K \sim\left(\begin{array}{ccc}
* & * & -t_{31} t_{33} \\
* & * & -t_{32} t_{33} \\
* & * & -t_{33}^{2}
\end{array}\right)
$$

which implies $t_{31}=t_{32}=0$. Using again $K \sim T^{\top} K T$ we find

$$
K \sim\left(\begin{array}{ccc}
* & t_{12} t_{33} & 0 \\
* & * & 0 \\
0 & 0 & -t_{33}^{2}
\end{array}\right)
$$

from which we deduce $t_{12}=0$. Then, finally,

$$
K \sim\left(\begin{array}{ccc}
t_{33}^{2} & 0 & 0 \\
0 & t_{22}^{2} & 0 \\
0 & 0 & -t_{33}^{2}
\end{array}\right)
$$

Hence $t_{22}= \pm 1$ and w.l.o.g. we may choose $t_{33}=1$.

Lemma 2.7 If $T \in \mathfrak{G}_{K}$ has the fixed point $(0,0,1)^{\top}$ and

$$
T(1,0,1)^{\top}=(\cos \phi, \sin \phi, 1)^{\top},
$$

then $T=D_{\phi}$ or $T=D_{\phi} S=: S_{\phi}$, where $S=\operatorname{diag}(1,-1,1)$ and

$$
D_{\phi}:=\left(\begin{array}{ccc}
\cos \phi & -\sin \phi & 0 \\
\sin \phi & \cos \phi & 0 \\
0 & 0 & 1
\end{array}\right) .
$$

Proof Because of Lemma 2.6, we have $D_{\phi}^{-1} T=\mathbb{I}$ or $D_{\phi}^{-1} T=S$.

Lemma 2.8 If the projective map $T \in \mathfrak{G}_{K}$ has fixed point $(0,0,1)^{\top}$ and $T(\cos \phi, \sin \phi, 1)^{\top}=(\cos \psi, \sin \psi, 1)^{\top}$, then $T=D_{\psi} D_{\phi}^{-1}=D_{\psi-\phi}$ or $T=$ $S_{\psi} D_{\phi}^{-1}=S_{\psi+\phi}$

Proof The projective map $T D_{\phi} \in \mathfrak{G}_{K}$ has the fixed point $(0,0,1)^{\top}$, and $T D_{\phi}(1,0,1)^{\top}=(\cos \psi, \sin \psi, 1)^{\top}$. Therefore, by Lemma 2.7, $T D_{\phi}=D_{\psi}$ or $T D_{\phi}=S_{\psi}$. 
Summarizing, we have the following: If $T \neq \mathrm{id}$ is a projective map with fixed point $(0,0,1)^{\top}$ which maps the unit circle $K$ to itself, then $T$ is either a rotation $D_{\eta}$ (namely if $(0,0,1)^{\top}$ is the only fixed point) or $T$ is a reflection $S_{\eta}$. In particular: If $T \in \mathfrak{G}_{K}$ has the fixed points $(0,0,1)^{\top}$ and $(1,0,1)^{\top}$, then $T$ is either the identity or the reflection with respect to the line $(0,1,0)^{\top}$.

A conic $C$ divides the points $\mathbb{P}$ of the projective plane into three disjoint sets: The points on $C$ (for those points there is exactly one tangent to $C$ ), the exterior points (for those points there are two tangents to $C$ ) and the inner points (which are not incident with a tangent of $C$ ). Observe, that this classification is invariant under projective maps. In particular, if $T \in \mathfrak{G}_{K}$ and if $z$ is an inner point of $K$, then $T z$ is also an inner point of $K$. For example, with

$$
U_{\psi}:=\left(\begin{array}{ccc}
\cosh \psi & 0 & \sinh \psi \\
0 & 1 & 0 \\
\sinh \psi & 0 & \cosh \psi
\end{array}\right) \in \mathfrak{G}_{K}
$$

we have $U_{\psi}(0,0,1)^{\top} \sim(\tanh \psi, 0,1)^{\top}$.

Let $T \in \mathfrak{G}_{K}$ and $T(0,0,1)^{\top}=(\tanh \psi \cos \phi, \tanh \psi \sin \phi, 1)^{\top}$ be an arbitrary inner point of $K$. Then $U_{\psi}^{-1} D_{\phi}^{-1} T \in \mathfrak{G}_{K}$ has the fixed point $(0,0,1)^{\top}$ and is hence either a rotation $D_{\eta}$ or a reflection $S_{\eta}$. Therefore, $T=D_{\phi} U_{\psi} D_{\eta}$ or $T=D_{\phi} U_{\psi} S_{\eta}$. Thus, we have:

Theorem 2.9 The group $\mathfrak{G}_{K}$ is generated by

$$
\left(\begin{array}{ccc}
\cos \phi & -\sin \phi & 0 \\
\sin \phi & \cos \phi & 0 \\
0 & 0 & 1
\end{array}\right), \quad\left(\begin{array}{ccc}
\cosh \psi & 0 & \sinh \psi \\
0 & 1 & 0 \\
\sinh \psi & 0 & \cosh \psi
\end{array}\right),\left(\begin{array}{ccc}
1 & 0 & 0 \\
0 & -1 & 0 \\
0 & 0 & 1
\end{array}\right)
$$

where $\phi \in[0,2 \pi[, \psi \in]-\infty, \infty[$.

For later use we add the following two lemmata:

Lemma 2.10 Let $K=\operatorname{diag}(1,1,-1)$ be the affine unit circle and $g$ a line which misses $K$. Then, $g$ can be written as $g=(\tanh \psi \cos \phi, \tanh \psi \sin \phi, 1)^{\top}$ for some $\psi \geq 0, \phi \in\left[0,2 \pi\left[\right.\right.$, and $T=U_{-\psi}^{-1} D_{\phi}^{-1} \in \mathfrak{G}_{K}$ maps $g$ to the ideal line $(0,0,1)$.

Proof The pole $P=K^{-1} g \sim(\tanh (-\psi) \cos \phi, \tanh (-\psi) \sin \phi, 1)^{\top}$ of $g$ with respect to $K$ is an inner point of $K$ and $T=U_{-\psi}^{-1} D_{\phi}^{-1} \in \mathfrak{G}_{K}$ maps $P$ to the origin, and hence $g$ to the ideal line.

Lemma 2.11 Let $P_{1}, P_{2}, P_{3}$ and $Q_{1}, Q_{2}, Q_{3}$ be two triples of points on the affine unit circle $K$. Then, there is a projective map $T \in \mathfrak{G}_{K}$ which maps $P_{i}$ to $Q_{i}, i \in\{1,2,3\}$.

Proof It suffices to show the lemma for $Q_{1}=(1,0,1)^{\top}, Q_{2}=(-1,0,1)^{\top}, Q_{3}=$ $(0,1,1)^{\top}$. By using a suitable rotation $D_{\phi}$ we may assume that $P_{1}$ and $P_{2}$ have the same $x_{1}$ component: $P_{1,2}=(\cos \phi, \pm \sin \phi, 1)^{\top}$. Then, $U_{\psi}$ maps $P_{1,2}$ to the points $(0, \pm 1,1)^{\top}$ for $\psi=-\operatorname{artanh} \cos \phi$. Another rotation maps these points to $Q_{1}=(1,0,1)^{\top}$ and $Q_{3}=(-1,0,1)^{\top}$, and the image of $P_{3}$ under the previous 
operations is some point $(\cos \eta, \sin \eta, 1)^{\top}$. Then, a suitable map $U_{\xi}$ maps this point to either $(0,1,1)^{\top}$ or $(0,-1,1)^{\top}$ and leaves the points $Q_{1}$ and $Q_{2}$ invariant. If necessary, a reflection $S$ with respect to the $x_{1}$ axis completes the proof.

The circle $\left(x_{1}-a_{1} x_{3}\right)^{2}+\left(x_{2}-a_{2} x_{3}\right)^{2}-r^{3} x_{3}^{2}=0$ with radius $r$ and center $\left(a_{1}, a_{2}\right)$ in the affine plane corresponds to the matrix

$$
\left(\begin{array}{ccc}
1 & 0 & -a_{1} \\
0 & 1 & -a_{2} \\
-a_{1} & -a_{2} & a_{1}^{2}+a_{2}^{2}-r^{2}
\end{array}\right)
$$

Vice versa, the conic

$$
\left(\begin{array}{lll}
1 & 0 & \alpha \\
0 & 1 & \beta \\
\alpha & \beta & \gamma
\end{array}\right)
$$

corresponds to a circle with center $(-\alpha,-\beta)$ and radius $r^{2}=\alpha^{2}+\beta^{2}-\gamma$ if this number is strictly positive.

For two different circles

$$
K_{i}=\left(\begin{array}{ccc}
1 & 0 & -a_{i} \\
0 & 1 & -b_{i} \\
-a_{i} & -b_{i} & a_{i}^{2}+b_{i}^{2}-r_{i}^{2}
\end{array}\right)
$$

$i \in\{1,2\}$, the difference

$$
K_{1}-K_{2}=\left(\begin{array}{ccc}
0 & 0 & a_{2}-a_{1} \\
0 & 0 & b_{2}-b_{1} \\
a_{2}-a_{1} & b_{2}-b_{1} & a_{2}^{2}-a_{1}^{2}+b_{2}^{2}-b_{1}^{2}+r_{2}^{2}-r_{1}^{2}
\end{array}\right)
$$

is a matrix of rank $<3$ and represents two lines

$$
\left\langle x,\left(K_{1}-K_{2}\right) x\right\rangle=\left\langle\left(\begin{array}{l}
0 \\
0 \\
1
\end{array}\right), x\right\rangle\left\langle\left(\begin{array}{c}
2\left(a_{2}-a_{1}\right) \\
2\left(b_{2}-b_{1}\right) \\
a_{1}^{2}-a_{2}^{2}+b_{1}^{2}-b_{2}^{2}+r_{2}^{2}-r_{1}^{2}
\end{array}\right), x\right\rangle .
$$

The line that corresponds to the first factor is the ideal line, the second line is the radical axis of the two circles (which is different from the ideal line if $K_{1}$ and $K_{2}$ have different centers). Vice versa, we have:

Theorem 2.12 Let $K$ be the affine unit circle

$$
K=\left(\begin{array}{ccc}
1 & 0 & 0 \\
0 & 1 & 0 \\
0 & 0 & -1
\end{array}\right)
$$


and $A$ a conic. Suppose, the pencil $K+\lambda A$ of $K$ and $A$ contains the ideal line $(0,0,1)^{\top}$, then $A$ is a circle.

Proof Let $e=(0,0,1)^{\top}$. Then, for certain $\lambda, \mu \in \mathbb{R}$ and a line $g$, the quadratic form

$$
\langle x,(K+\lambda A) x\rangle=2 \mu\langle e, x\rangle\langle g, x\rangle=2 \mu x_{3}\left(g_{1} x_{1}+g_{2} x_{2}+g_{3} x_{3}\right)
$$

is represented by

$$
K+\lambda A=\mu\left(\begin{array}{ccc}
0 & 0 & g_{1} \\
0 & 0 & g_{2} \\
g_{1} & g_{2} & 2 g_{3}
\end{array}\right)
$$

Obviously, $\lambda \neq 0$ and

$$
A \sim\left(\begin{array}{ccc}
1 & 0 & -\mu g_{1} \\
0 & 1 & -\mu g_{2} \\
-\mu g_{1} & -\mu g_{2} & -1-2 \mu g_{3}
\end{array}\right) .
$$

which is a circle in the affine plane with radius $r^{2}=1+2 \mu g_{3}+\mu^{2}\left(g_{1}^{2}+g_{2}^{2}\right)$ and center $\mu\left(g_{1}, g_{2}\right)$.

As a consequence of Lemma 2.10 and Theorem 2.12 we get:

Corollary 2.13 Let $K$ be the unit circle and A a conic such that the pencil of $K$ and A contains a line $g$ which misses both $K$ and A. Then, the projective map $T$ which leaves $K$ invariant and sends $g$ to the ideal line maps $A$ to a circle.

Proof According to Lemma 2.10 there exists a map $T \in \mathfrak{G}_{K}$ which sends $g$ to the ideal line. Observe that $T$ maps the pencil of $K$ and $A$ to the pencil of their images, $K$ and $\tilde{T}:=T^{-\top} A T^{-1}$. Therefore, the ideal line belongs to the pencil of $K$ and $\tilde{T}$ and thus, according to Theorem $2.12, \tilde{T}$ is a circle.

\section{How to transform two conics into two circles}

Let us first recall, how one can find coordinates for which one given conic is a circle. For this, consider a conic $A \in \mathbb{R}^{3 \times 3}$, symmetric, regular and with mixed signature. The signature of $A$ can be determined from the characteristic polynomial:

Lemma 3.1 Let $A \in \mathbb{R}^{3 \times 3}$ be regular and symmetric with characteristic polynomial $p_{A}(\lambda)=\operatorname{det}(A-\lambda I)=a_{0}+a_{1} \lambda+a_{2} \lambda^{2}-\lambda^{3}$. Then the following holds:

- If all eigenvalues of $A$ are positive, we have $a_{0}>0, a_{1}<0, a_{2}>0$.

- If all eigenvalues of $A$ are negative, we have $a_{0}<0, a_{1}<0, a_{2}<0$.

- If $A$ has two negative and one positive eigenvalue, then $a_{0}>0$, and if $a_{1}<0$ then $a_{2}<0$.

- If A has two positive and one negative eigenvalue, then $a_{0}<0$, and if $a_{1}<0$ then $a_{2}>0$. 
Proof Since $A$ is symmetric, all eigenvalues $\lambda_{1}, \lambda_{2}, \lambda_{3}$ are real and $p_{A}(\lambda)=\left(\lambda_{1}-\right.$ $\lambda)\left(\lambda_{2}-\lambda\right)\left(\lambda_{3}-\lambda\right)$. Hence, $a_{0}=\lambda_{1} \lambda_{2} \lambda_{3} \neq 0, a_{1}=-\left(\lambda_{1} \lambda_{2}+\lambda_{1} \lambda_{3}+\lambda_{2} \lambda_{3}\right)$ and $a_{2}=\lambda_{1}+\lambda_{2}+\lambda_{3}$. It is then easy to check the four cases.

The transformation of $A$ to a unit circle is then as follows:

Lemma 3.2 Let $A \in \mathbb{R}^{3 \times 3}$ be a conic. Without loss of generality, A has two positive and one negative eigenvalue (otherwise take the representative $-A$ ). Then there is a regular matrix $T$ such that $T^{\top} A T=\operatorname{diag}(1,1,-1)$. This corresponds to the unit circle in the affine plane.

Proof Let $\lambda_{1}, \lambda_{2}>0$ be the positive eigenvalues, $\lambda_{3}<0$ the negative eigenvalue, and $x_{1}, x_{2}, x_{3}$ be the corresponding orthonormal eigenvectors. Then the matrix $T$ with columns $\frac{1}{\sqrt{\lambda_{1}}} x_{1}, \frac{1}{\sqrt{\lambda_{2}}} x_{2}, \frac{1}{\sqrt{-\lambda_{3}}} x_{3}$ has the desired property.

In his seminal work Poncelet (1822)[Section I, Chapitre III, no. 121, p. 59] Poncelet claims that two conics can, in general, be considered as projective image of two circles. Actually, referring to this statement, he reduces the proof of Poncelet's Porism to the case of a pencil of circles in Poncelet (1822)[Section IV, Chapitre II, no. 530, p. $311 \mathrm{ff}$.]. The question whether two conics are the projective image of two circles also occurs in Questions proposées (1816-1817) as a proposed problem, with alleged solution in Par un Abonné (1816-1817). However, it is clear that for example two conics which intersect in four points cannot be the projective image of two circles.

It turns out that two conics can lie in 8 different positions relative to each other (see Petitjean 2010):

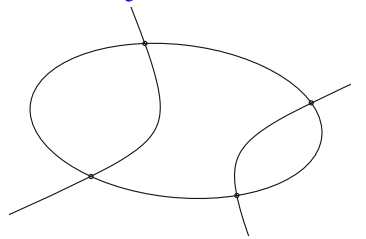

Case 1: four intersections

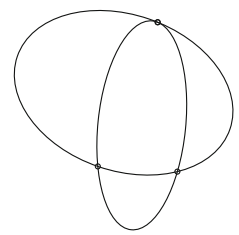

Case 4: two intersections, one 1st order contact

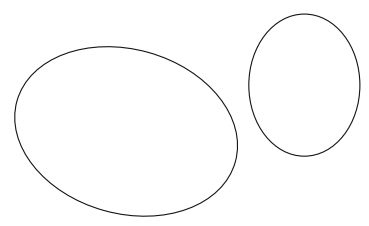

Case 2: no intersections

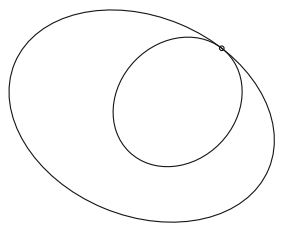

Case 5: one 1st order contact contacts

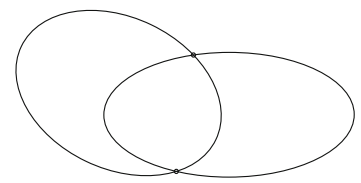

Case 3: two intersections

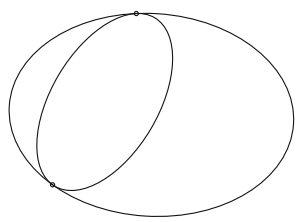

Case 6: two 1st order

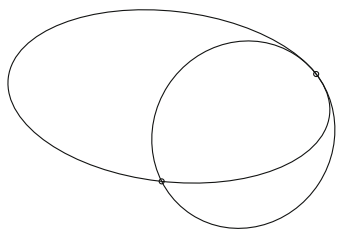

Case 7: one intersection, one Case 8: one 3rd order contact 2nd order contact 
Projective transformations do not change the number of intersections nor the order of contact, hence the cases $1,4,6,7$ and 8 cannot be the projective image of two circles. In fact we have:

Theorem 3.3 Two conics are the projective image of two circles if and only if they (i) intersect in two points, (ii) have one 1st order contact, or (iii) are disjoint.

Proof By the remark above, we only need to show one implication. Without loss of generality, we may assume that one of the conics is the circle $A=\operatorname{diag}(1,1,-1)$. $B \in \mathbb{R}^{3 \times 3}$ is an arbitrary conic different from $A$. According to Corollary 2.13 , it suffices to show in each of the three cases (i)-(iii) that the pencil of $A$ and $B$ contains a line that misses both conics.

(i) Suppose $A$ and $B$ intersect in two points $z_{1}, z_{2}$. The line $g=z_{1} \times z_{2}$ is the common secant of $A$ and $B$. Let $p$ be a point on $g$ different from $z_{1}, z_{2}$ and

$$
r=-\frac{\langle p, A p\rangle}{\langle p, B p\rangle} \neq 0
$$

Then, $z_{1}, z_{2}$ and $p$ are solutions of $\langle x,(A+r B) x\rangle=0$. Since $z_{1}, z_{2}$ and $p$ are collinear, we conclude $\operatorname{rank}(A+r B)<3$. On the other hand, $A$ and $B$ are different conics, hence $\operatorname{rank}(A+r B)>0$. Moreover, $\operatorname{rank}(A+r B) \neq 1$, because otherwise, $-A$ and $-A+(A+r B)=r B$ would be tangential in $z_{1}$ and $z_{2}$ (see Lemma 2.5). Thus, $\operatorname{rank} A=2$ and by Lemma 2.4, we have $A+r B=\alpha a a^{\top}+\beta b b^{\top}$ for two orthonormal vectors $a, b$ and $\alpha, \beta \in \mathbb{R}^{3} \backslash\{0\}$. If the eigenvalues $\alpha, \beta$ would both be positive or both be negative, $\langle x,(A+r B) x\rangle=$ 0 would have only one solution (see Lemma 2.4), but we have at least three, namely $z_{1}, z_{2}, p$. Therefore, $A+r B$ has mixed signature and hence, according to Lemma 2.4, $A+r B=u v^{\top}+v u^{\top}$ for two non-zero vectors $u \neq v \in \mathbb{R}^{3}$, and $\langle x,(A+r B) x\rangle=2\langle x, u\rangle\langle x, v\rangle=0$ consists of two lines: One of them is $g=u$, the other, $v \neq u$, has no common point with the conics $A$ and $B$ : Indeed, suppose we have a point $p$ on $v$ and $A$ or $B$, then $p$ would solve $\langle p, A p\rangle=0$ and $\langle p, B p\rangle=0$. Hence, w.l.o.g., $p=z_{1}$. But then, $v$ cuts $A$ or $B$ in a second point, which is necessarily $z_{2}$. This contradics $u \neq v$.

(ii) Suppose, $A$ and $B$ have one contact point, say $z_{0}$, in common. Let $p \neq z_{0}$ be a point on the tangent $g$ in $z_{0}$ and $r$ as above in (2). Then, $p$ and $z_{0}$ are solutions of $\langle x,(A+r B) x\rangle=0$. Suppose $A+r B$ has full rank. Then, the tangent $g$ in $z_{0}$ at the conic $A+r B$ does not contain another point of that same conic. But this is not true, since $p$ is a point of $g$. Hence, $\operatorname{rank}(A+r B)<3$. Since, by assumption, $A$ and $B$ are different conics, $\operatorname{rank}(A+r B)>0$. Suppose, $\operatorname{rank}(A+r B)=1$. We may assume, that $z_{0}=(0,1,1)^{\top}$ and thus $g=(0,-1,1)^{\top}$. According to Lemma 2.4 $A+r B \sim a a^{\top}$ for a unit vector $a$. Hence, $g \sim(A+r B) z_{0} \sim a a^{\top} z_{0} \sim a$, and we conclude

$$
B \sim A+\mu g g^{\top} \sim A+\mu\left(\begin{array}{ccc}
0 & 0 & 0 \\
0 & 1 & -1 \\
0 & -1 & 1
\end{array}\right) \sim\left(\begin{array}{ccc}
1 & 0 & 0 \\
0 & \mu+1 & -\mu \\
0 & -\mu & \mu-1
\end{array}\right)
$$


and $\langle x, B x\rangle=x_{1}^{2}+x_{2}^{2}(1+\mu)-x_{3}^{2}(1-\mu)-2 x_{2} x_{3} \mu$. It is then easy to check, that $A$ and $B$ have a 3 rd order contact in $z_{0}$, which, by assumption, is not the case. Therefore, $\operatorname{rank}(A+r B)=2$, and, by the same reasoning as above in (i), we conclude, that $\langle x,(A+r B) x\rangle=2\langle x, g\rangle\langle x, v\rangle=0$ consists of two lines, $g \neq v$, and that $v$ has no common point with $A$ or $B$.

(iii) Suppose, $A$ and $B$ are disjoint. Then, there exist coordinates for which both conics are diagonal (see for example Pesonen 1956 or Hong et al. 1986): W.l.o.g.

$$
A=\left(\begin{array}{ccc}
1 & 0 & 0 \\
0 & 1 & 0 \\
0 & 0 & -1
\end{array}\right), \quad B=\left(\begin{array}{ccc}
1 & 0 & 0 \\
0 & a & 0 \\
0 & 0 & b
\end{array}\right)
$$

where $a, b \neq 0$ are not both positive. Then, for the values $r \in\left\{-1,-\frac{1}{a}, \frac{1}{b}\right\}$, we have for $A+r B$ :

$$
\begin{gathered}
A-B=\left(\begin{array}{ccc}
0 & 0 & 0 \\
0 & 1-a & 0 \\
0 & 0 & -1-b
\end{array}\right), \quad A-\frac{1}{a} B=\left(\begin{array}{ccc}
1-\frac{1}{a} & 0 & 0 \\
0 & 0 & 0 \\
0 & 0 & -1-\frac{b}{a}
\end{array}\right) \\
A+\frac{1}{b} B=\left(\begin{array}{ccc}
1+\frac{1}{b} & 0 & 0 \\
0 & 1+\frac{a}{b} & 0 \\
0 & 0 & 0
\end{array}\right) .
\end{gathered}
$$

For all values of $a$ and $b$ one of these three matrices has mixed signature and is of rank 2 . The corresponding quadratic form $\langle x,(A+r B) x\rangle=2\langle x, g\rangle\langle x, h\rangle=0$ represents two lines which both miss $A$ and $B$.

\section{Weakly connected closed chains of Poncelet triangles}

Let us first recall the Cayley criterion for Poncelet polygons:

Theorem 4.1 (Cayley criterion, see Cayley 1854) Let $A, H$ be conics, $D(\lambda)=$ $\operatorname{det}(A+\lambda H)$, and

$$
\sqrt{D(\lambda)}=c_{0}+c_{1} \lambda+c_{2} \lambda^{2}+c_{3} \lambda^{3}+\cdots
$$

Then, there exists a Poncelet $k$-gon with vertices on $H$ and tangent to $A$ if and only if

$$
\operatorname{det}\left(\begin{array}{cccc}
c_{3} & c_{4} & \ldots & c_{p+1} \\
c_{4} & c_{5} & \ldots & c_{p+2} \\
\ldots & & & \\
c_{p+1} & c_{p+2} & \ldots & c_{2 p-1}
\end{array}\right)=0 \text { for } k=2 p
$$


or

$$
\operatorname{det}\left(\begin{array}{cccc}
c_{2} & c_{3} & \ldots & c_{p+1} \\
c_{3} & c_{4} & \ldots & c_{p+2} \\
\ldots & & & \\
c_{p+1} & c_{p+2} & \ldots & c_{2 p}
\end{array}\right)=0 \text { for } k=2 p+1
$$

In this section, we will consider chains of conics, where each pair of consecutive conics is a Poncelet pair for triangles. The main result of this section is that every such chain can be closed. Let us start with the following definition:

A chain of conics $G_{0}, G_{1}, \ldots, G_{n}, n \geq 1$, is called weakly connected Poncelet chain for triangles if, for $i \in\{0,1, \ldots, n-1\},\left(G_{i}, G_{i+1}\right)$ is a Poncelet pair for triangles, i.e., $G_{i}$ carries verticies of triangles whose sides are tangential to $G_{i+1}$. The chain is called closed and of length $n$, if $G_{n}=G_{0}$.

The central lemma which allows to close a chain is the following:

Lemma 4.2 Let $G$ and $H$ be conics. Then, there exists a conic $A$ such that $(H, A)$ and $(A, G)$ are Poncelet pairs for triangles: $H$ carries vertices of triangles that are tangent to $A$, and $A$ carries vertices of triangles that are tangent to $G$.

We call $A$ a lock between $G$ and $H$.

The six coefficients of $A$ have to satisfy two polynomial equations of oder 2 and 4 respectively (the Cayley conditions), $A$ needs to have mixed signature, and it has to have points outside of $G$ and inside of $H$.

Proof of Lemma 4.2 We have to consider the 8 cases listed in Sect. 3.

Case 1. $G$ and $H$ have four intersections $P_{1}, P_{2}, P_{3}, P_{4}$. By Lemma 3.2 we may assume, that $G$ is the unit circle $K$. Let $P$ be the intersection of the lines $P_{1} P_{3}$ and $P_{2} P_{4}$ joining opposite points. Then we choose $T \in \mathfrak{G}_{K}$ such that $T$ maps $P$ to $(0,0,1)^{\top}$ (see Theorem 2.9). Thus, the image of $H$ is a conic with center $(0,0,1)^{\top}$, either a hyperbola or an ellipse. By a suitable rotation $D_{\phi}$, we may further assume that $H$ is symmetric with respect to the $x_{1}$ - and $x_{2}$-axis.

Case 1a. A circle and a concentric hyperbola:

$$
G=\left(\begin{array}{ccc}
1 & 0 & 0 \\
0 & 1 & 0 \\
0 & 0 & -1
\end{array}\right) \quad \text { and } H=\left(\begin{array}{ccc}
a^{2} & 0 & 0 \\
0 & -b^{2} & 0 \\
0 & 0 & -1
\end{array}\right)
$$

We choose

$$
A=\left(\begin{array}{ccc}
0 & x & x \\
x & 1 & -1 \\
x & -1 & 1
\end{array}\right) \quad \text { with } \quad x=\frac{4 a}{\sqrt{b^{2}+1}}
$$

Then a direct computation shows that

$$
\left.\frac{d^{2}}{d \lambda^{2}} \sqrt{\operatorname{det}(G+\lambda A)}\right|_{\lambda=0}=0 \text { and }\left.\frac{d^{2}}{d \lambda^{2}} \sqrt{\operatorname{det}(A+\lambda H)}\right|_{\lambda=0}=0
$$


which shows that the Cayley criterion in Theorem 4.1 for triangles is satisfied for both pairs $H, A$ and $A, G$.

The eigenvalues of $A$ are $\{2, \sqrt{2} x,-\sqrt{2} x\}$, i.e., $A$ has mixed signature. As $A$ is a hyperbola, it has points outside $G$. A passes through the point $(0,1,1)$ and has the asymptote $(0,1,1)^{\top}$. Therefore, the intersections of this asymptote with $H$ are outer points of $A$.

Case 1b. A circle and a concentric ellipse:

$$
G=\left(\begin{array}{ccc}
1 & 0 & 0 \\
0 & 1 & 0 \\
0 & 0 & -1
\end{array}\right) \quad \text { and } \quad H=\left(\begin{array}{ccc}
a^{2} & 0 & 0 \\
0 & b^{2} & 0 \\
0 & 0 & -1
\end{array}\right), b^{2} \neq 1
$$

We choose

$$
A=\left(\begin{array}{lll}
y & x & x \\
x & y & 0 \\
x & 0 & 0
\end{array}\right)
$$

with

$$
x=\sqrt{1+2 a^{2}+b^{2}+2 \sqrt{\left(1+a^{2}\right)\left(a^{2}+b^{2}\right)}}, \quad y=b^{2}-1 .
$$

Again, the Cayley criterion (3) for triangles is easily verified. By Lemma 3.1 it can be checked, that $A$ has mixed signature. In fact, $A$ is a hyperbola through $(0,0,1)^{\top}$ and has therefore points outside $G$, and $H$ has points outside $A$.
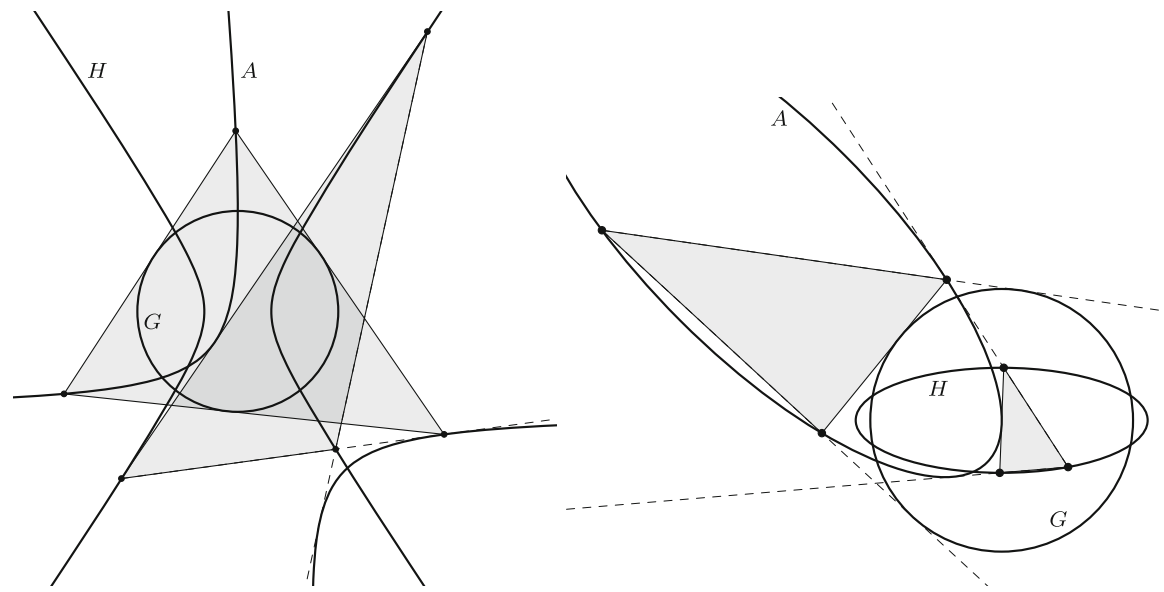

Lock in Case 1a (left) and 1b (right): $H$ carries vertices of Poncelet triangles that are tangent to $A, A$ carries vertices of Poncelet triangles that are tangent to $G$

Cases 2 and 3. $G$ and $H$ have either no common points or two intersections. By Theorem 3.3, we may assume, that $G$ is the affine unit circle, and $H$ another circle having its center on the $x_{1}$-axis: 


$$
G=\left(\begin{array}{ccc}
1 & 0 & 0 \\
0 & 1 & 0 \\
0 & 0 & -1
\end{array}\right) \text { and } H=\left(\begin{array}{ccc}
1 & 0 & -a \\
0 & 1 & 0 \\
-a & 0 & a^{2}-r^{2}
\end{array}\right) \text { with } a \geq 0
$$

We choose

$$
A=\left(\begin{array}{lll}
0 & 0 & x \\
0 & 1 & 0 \\
x & 0 & y
\end{array}\right)
$$

with

$$
x=\sqrt{1+(1+a-r)^{2}}+r-1-a, \quad y=2 \sqrt{1+(1+a-r)^{2}}+2 r-3-2 a .
$$

Then the Cayley criterion (3) for triangles is easily verified. $A$ is a parabola symmetric to the $x_{1}$-axis with vertex inside $H$ and has therefore points outside $G$, and $H$ has points outside $A$.

Case 4. $G$ and $H$ have two intersections and one first order contact. We may again assume that $G$ is the affine unit circle $\operatorname{diag}(1,1,-1)$, and, by Lemma 2.11 , that the first order contact is in $(1,0,1)^{\top}$ and that the two intersections are $(0, \pm 1,1)^{\top}$. Then, the pencil of $G$ and $H$ contains two lines $(1, \pm 1,-1)^{\top}$, and according to Lemma 2.4

$$
H=G+\mu\left(\begin{array}{ccc}
1 & 0 & -1 \\
0 & -1 & 0 \\
-1 & 0 & 1
\end{array}\right)=\left(\begin{array}{ccc}
\mu+1 & 0 & -\mu \\
0 & 1-\mu & 0 \\
-\mu & 0 & \mu-1
\end{array}\right), \mu \notin\{0,1\}
$$

We choose

$$
A=\left(\begin{array}{lll}
0 & 0 & p \\
0 & 1 & 0 \\
p & 0 & q
\end{array}\right)
$$

with

$$
p=\frac{2 \mu-\sqrt{1+3 \mu^{2}}}{\mu-1}, \quad q=\frac{1+3 \mu-2 \sqrt{1+3 \mu^{2}}}{\mu-1} .
$$

A direct calculation shows that the Cayley criterion (3) is satisfied. $A$ is a parabola which is symmetric to the $x_{1}$-axis. Its vertex $\left(x_{1}, 0,1\right)^{\top}$ satisfies $x_{1}<1$. In particular, $A$ has points outside $G$. $H$ is also symmetric to the $x_{1}$-axis: For $\mu<-1$ and $\mu>1$ it is a hyperbola, for $-1<\mu<1$ an ellipse, and for $\mu=-1$ a parabola. In each case, $H$ has points outside $A$.

Case 5. $G$ and $H$ have one first order contact: see Cases 2 and 3.

Case 6. $G$ and $H$ have two first order contacts. As above, we may assume, that $G$ is the affine unit circle $\operatorname{diag}(1,1,-1)$, and, by Lemma 2.11 , that the contact points are 
$( \pm 1,0,1)^{\top}$. The pencil of $G$ and $H$ contains the double line $(0,1,0)^{\top}$ and according to Lemma 2.4:

$$
H=G-\mu\left(\begin{array}{lll}
0 & 0 & 0 \\
0 & 1 & 0 \\
0 & 0 & 0
\end{array}\right)=\left(\begin{array}{ccc}
1 & 0 & 0 \\
0 & 1-\mu & 0 \\
0 & 0 & -1
\end{array}\right), \mu \neq 0
$$

If $\mu>0$, we choose

$$
A=\left(\begin{array}{ccc}
0 & q & q \\
q & 1 & -1 \\
q & -1 & 1
\end{array}\right) \text { with } q=\frac{4}{\sqrt{\mu}}
$$

If $\mu<0$, we choose

$$
A=\left(\begin{array}{ccc}
p & 0 & 0 \\
0 & 0 & -1 \\
0 & -1 & 2-q
\end{array}\right) \text { with } p=1-\frac{1}{\sqrt{1-\mu}}+\sqrt{\frac{\mu-3+2 \sqrt{1-\mu}}{\mu-1}}
$$

In both cases, it is easy to check, that the Cayley criterion (3) is satisfied, that $A$ has mixed signature and has points outside $G$. Moreover, $H$ has points outside $A$.

Case 7. $G$ and $H$ have one intersection and one first order contact. We may again assume, that $G$ is the affine unit circle $K$. By Lemma 2.11 we may assume, that the first order contact is in $(1,0,1)^{\top}$ and the intersection in $(-1,0,1)^{\top}$. Then the lines $(-1,0,1)^{\top}$ and $(0,1,0)^{\top}$ belong to the pencil of $G$ and $H$ and according to Lemma 2.4:

$$
H=K-\mu\left(\begin{array}{ccc}
0 & -1 & 0 \\
-1 & 0 & 1 \\
0 & 1 & 0
\end{array}\right)=\left(\begin{array}{ccc}
1 & -\mu & 0 \\
-\mu & 1 & \mu \\
0 & \mu & -1
\end{array}\right), \mu \neq 0
$$

Then, the choice

$$
A=\left(\begin{array}{ccc}
0 & q & q \\
q & 1 & -1 \\
q & -1 & 1
\end{array}\right) \text { with } q=\frac{2}{\mu}\left(2+\mu+\sqrt{4-2 \mu+\mu^{2}}\right)
$$

yields the desired lock.

Case 8. $G$ and $H$ have one third order contact. We may assume that $G$ is the affine unit circle $(1,1,-1)^{\top}$, and, by a suitable rotation, that the point of contact is $(0,1,1)^{\top}$. As discussed in the proof of Theorem 3.3 the pencil of $G$ and $H$ contains the double line $(0,-1,1)^{\top}$. Thus, by Lemma 2.4 ,

$$
H=G+\mu\left(\begin{array}{ccc}
0 & 0 & 0 \\
0 & 1 & -1 \\
0 & -1 & 1
\end{array}\right)=\left(\begin{array}{ccc}
1 & 0 & 0 \\
0 & \mu+1 & -\mu \\
0 & -\mu & \mu-1
\end{array}\right)
$$


Here, if $\mu \neq-1$, we choose

$$
\begin{aligned}
A & =\left(\begin{array}{ccc}
1 & 0 & a \\
0 & -1 & b \\
a & b & 0
\end{array}\right) \text { with } a=\frac{\sqrt{1+\mu+4 \mu^{2}}-2 \mu}{1+\mu}, \\
b & =\frac{2 \mu}{1+\mu}-\frac{\sqrt{1+\mu+4 \mu^{2}}}{1+\mu}-2
\end{aligned}
$$

and, if $\mu=-1$,

$$
A=\left(\begin{array}{ccc}
1 & 0 & -1 \\
0 & -1 & 0 \\
-1 & 0 & 0
\end{array}\right)
$$

Using the above lemma, we are now able to prove the following

Theorem 4.3 Let $G_{0}, G_{1}, \ldots, G_{n-2}$ be a weakly connected Poncelet chain for triangles. Then, there exists a conic $G_{n-1}$ such that $G_{0}, G_{1}, \ldots, G_{n-1}, G_{n}=G_{0}$ is a closed weakly connected Poncelet chain for triangles.

Proof Choose $G_{n-1}$ to be a lock between $G_{n-2}$ and $G_{0}$, as described in Lemma 4.2.

As an immediate consequence we get

Corollary 4.4 There are closed weakly connected Poncelet chains for triangles of arbitrary length $n \geq 2$.

Proof For $n \geq 3$, this follows directly form Theorem 4.3. For $n=2$, the two conics

$$
G_{0}=\left(\begin{array}{ccc}
1 & 0 & \sqrt{3} / 2 \\
0 & 1 & 0 \\
\sqrt{3} / 2 & 0 & -1 / 4
\end{array}\right), \quad G_{1}=\left(\begin{array}{ccc}
1 & 0 & -\sqrt{3} / 2 \\
0 & 1 & 0 \\
-\sqrt{3} / 2 & 0 & -1 / 4
\end{array}\right)
$$

form a closed chain of length 2 .

\section{Rigid closed chains of Poncelet triangles}

In a closed weakly connected Poncelet chain $G_{0}, G_{1}, \ldots, G_{n-1}$ of length $n$ for triangles, as seen in Sect. 4, the Poncelet triangles $\Delta_{i}$ sitting between two consecutive conics $\left(G_{i}, G_{i+1}\right)$ of the chain are not related to the neighbouring triangles. In this section, we will require, that the contact points of the sides of $\Delta_{i}$ on $G_{i+1}$ are the vertices of the next triangle $\Delta_{i+1}$. Then, the problem of finding closed chains $G_{0}, \ldots, G_{n-1}$ with a chain of Poncelet triangles $\Delta_{0}, \ldots, \Delta_{n-1}$ satisfying this additional requirement, becomes more subtle. 
A closed chain of triangles $\Delta_{0}, \ldots, \Delta_{n-1}$ of length $n$ is called a rigid chain of Poncelet triangles if there are conics $G_{0}, \ldots, G_{n-1}$ such that $\Delta_{i}$ has its vertices on $G_{i}$ and is tangent to $G_{i+1}$, where the contact points are the vertices of $\Delta_{i+1}$ (where we take indices modulo $n$ ).

If we move one of the triangles, say $\Delta_{0}$, such that two of its sides are still tangent to $G_{1}$, then, by Poncelet's Theorem, also the third side is tangent to $G_{1}$. However, the contact points of the new triangle, which are points on $G_{1}$, are not necessarily the vertices of a triangle whose sides are tangent to $G_{2}$. So, in general we cannot move a rigid chain of Poncelet triangles (i.e., the Poncelet triangles sit rigidly in the corresponding chain of conics).

By Corollary 4.4 we know that there are closed weakly connected Poncelet chains for triangles of arbitrary length; a similar result we get also for rigid closed chains of Poncelet triangles.

Theorem 5.1 There are rigid closed chains of Poncelet triangles of arbitrary length $n \geq 3$.

Proof We first give an example of a rigid chain of Poncelet triangles of length $n=3$.

Let

$$
G_{0}=\left(\begin{array}{lll}
1 & 0 & 0 \\
0 & 1 & 1 \\
0 & 1 & 0
\end{array}\right), \quad G_{1}=\left(\begin{array}{ccc}
1 & 0 & 0 \\
0 & -1 & 0 \\
0 & 0 & -1
\end{array}\right), \quad G_{2}=\left(\begin{array}{ccc}
1 & 0 & 0 \\
0 & 0 & -1 \\
0 & -1 & 1
\end{array}\right)
$$

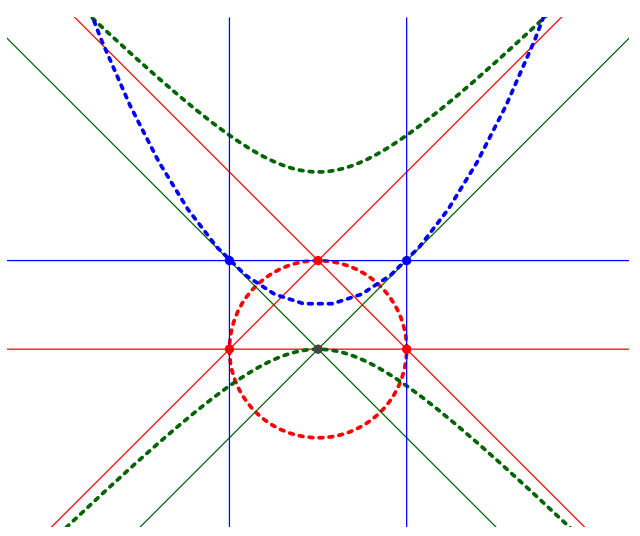

A rigid chain of Poncelet triangles of length $n=3$

It is not hard to see that the three triangles $\triangle A B C, \triangle P Q R, \triangle U V W$ on $G_{0}, G_{1}$, $G_{2}$, respectively, where 


$$
\begin{gathered}
A=\left(\begin{array}{l}
0 \\
0 \\
1
\end{array}\right) \quad B=\left(\begin{array}{c}
-1 \\
-1 \\
1
\end{array}\right) \quad C=\left(\begin{array}{c}
1 \\
-1 \\
1
\end{array}\right) \\
P=\left(\begin{array}{c}
0 \\
-1 \\
1
\end{array}\right) \quad Q=\left(\begin{array}{l}
1 \\
1 \\
0
\end{array}\right) \quad R=\left(\begin{array}{c}
1 \\
-1 \\
0
\end{array}\right) \\
U=\left(\begin{array}{l}
0 \\
1 \\
0
\end{array}\right) \quad V=\left(\begin{array}{c}
-1 \\
0 \\
1
\end{array}\right) \quad W=\left(\begin{array}{l}
1 \\
0 \\
1
\end{array}\right)
\end{gathered}
$$

form a rigid closed chain of Poncelet triangles of length $n=3$.

Now, if we enlarge the parabola and pinch the circle to an ellipse, we can plug in two additional ellipses between the parabola and the pinched circle in such a way, that the two additional triangles we get fit in the chain of pairwise Poncelet triangles. Notice that the hyperbola and the two triangles which are tangent to the hyperbola and the parabola respectively remain unchanged.

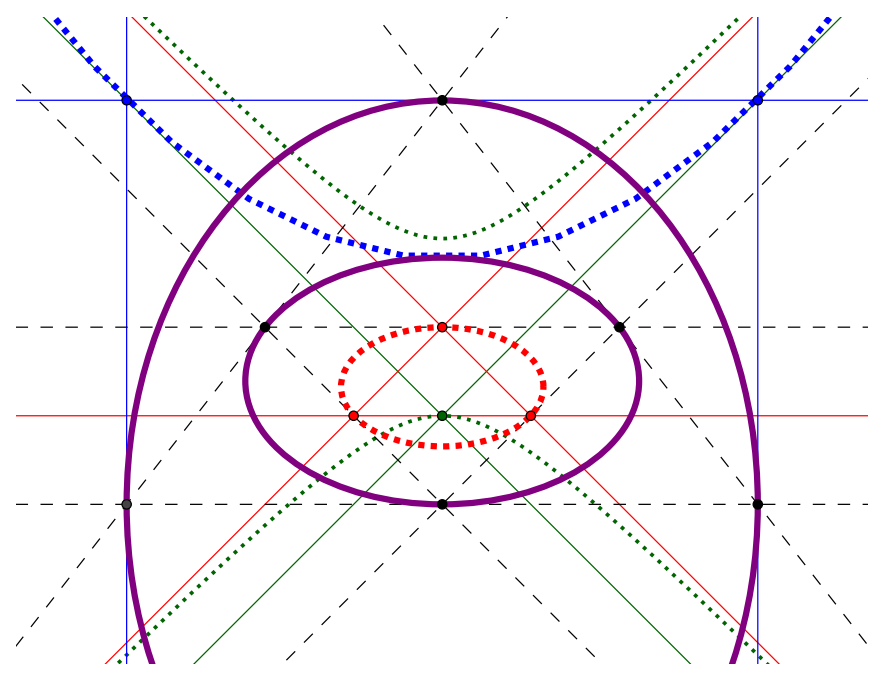

Two ellipses between the parabola and the pinched circle

By a similar construction, we can plug in as many pairs of ellipses as we like. So, we can construct rigid closed chains of Poncelet triangles of arbitrarily odd length $n \geq 3$.

In order to construct rigid chains of Poncelet triangles of even length, we proceed as follows: We start with the same closed chain of Poncelet triangles as above. Then we enlarge and reflect the parabola and pinch the circle to an ellipse, such that we can plug in an additional ellipse between the parabola and the pinched circle in such a way, that the additional triangle fits in the chain of pairwise Poncelet triangles. Notice 
that the hyperbola and the two triangles which are tangent to the hyperbola and the parabola respectively remain unchanged (see figure below).

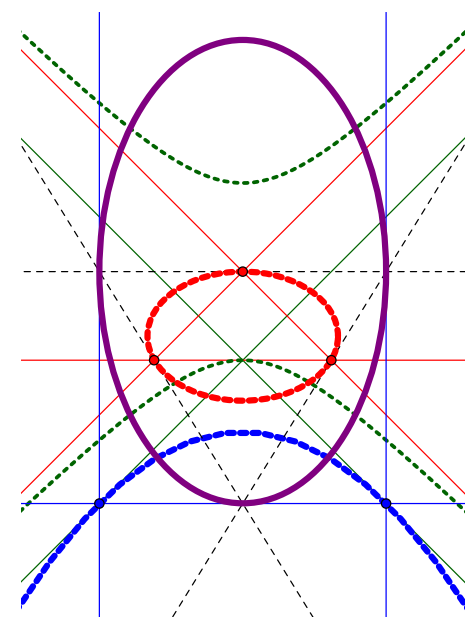

An ellipse between the reflected parabola and the pinched circle

Like above, we can now plug in arbitrarily many pairs of ellipses. So, we can construct rigid chains of Poncelet triangles of arbitrarily even length $n \geq 4$.

\section{Miraculous chains of Poncelet polygons}

In general, the triangles $\Delta_{i}$ in a closed rigid chain of Poncelet triangles satisfy the condition, that the contact points of $\Delta_{i}$ on $G_{i+1}$ are the vertices of $\Delta_{i+1}$, only in one particular position. In this section, we investigate the question, whether closed rigid chains exist such that the contact condition is satisfied in every position. For this, we first recall some relevant facts and notations.

If a point $x$ moves along a conic $G_{0}$, then each polar of $x$ with respect to a second conic $G_{1}$ is tangent to one particular conic $G_{2}$, which is called the conjugate of $\boldsymbol{G}_{\mathbf{0}}$ with respect to $\boldsymbol{G}_{\mathbf{1}}$ (see Halbeisen and Hungerbühler 2016 [Theorem 1.5]). In particular, if $K_{0}$ is a Poncelet $k$-gon, inscribed in $G_{0}$ and circumscribed about $G_{1}$, then the $k$-gon $K_{1}$ whose vertices are the contact points of $K_{0}$ on $G_{1}$ is tangent to the conjugate conic $G_{2}$ of $G_{0}$ with respect to $G_{1}$. Hence $K_{1}$ is itself a Poncelet $k$-gon for the pair $G_{1}$ and $G_{2}$. Obviously, this process can be iterated. Astonishingly, there are very particular configurations, where this process closes after a finite number of steps, i.e., the $n$-th $k$-gon $K_{n}$ is equal to $K_{0}$.

For an integer $k \geq 3$, a closed chain of pairwise conjugate conics $G_{0}, \ldots, G_{n-1}$ (i.e., $G_{i}$ and $G_{i+2}$ are conjugate with respect to $G_{i+1}$ ) is a miraculous chain for Poncelet $k$-gons if for each $0 \leq i<n, G_{i}$ carries the vertices of a $k$-gon $K_{i}$ which is tangent to $G_{i+1}$, where the contact points are the vertices of $K_{i+1}$ (where we take indices modulo $n$ ). 
Since we require that the conics $G_{0}, \ldots, G_{n-1}$ are pairwise conjugate, we can move the vertices of the $k$-gons without losing the property that the contact points of a $k$-gon are the vertices of the next $k$-gon.

We shall see that up to projective transformations, there is exactly one miraculous chain of Poncelet triangles and one miraculous chain for Poncelet quadrilaterals.

\subsection{Closed chains of conjugate conics}

From Halbeisen and Hungerbühler (2016) [Theorems 2.1 and 2.2]), we infer the following: Let $G_{0}, G_{1}, \ldots$ be a sequence of conics such that $G_{i+2}$ is the conjugate of $G_{i}$ with respect to $G_{i+1}$, for all indices $i \geq 0$. Then, $G_{i+2} \sim G_{1}\left(G_{0}^{-1} G_{1}\right)^{i+1}$.

Let $I$ denote the $3 \times 3$ identity matrix. A chain $G_{0}, G_{1}, G_{2}, \ldots, G_{n-1}, G_{n}=G_{0}$ of length $n$ of conjugate conics is closed iff there are representatives of the conics such that

$$
\left(G_{0}^{-1} G_{1}\right)^{n}=I .
$$

and $n$ is minimal with this property.

We now want to investigate, in which of the 8 cases listed in Sect. 3, closed chains may exist. For this, let $G_{0}, \ldots, G_{n-1}$, for some $n \geq 3$, be a closed chain of conjugate conics. We may assume, that $G_{0}, G_{1}$ are in standard form, as in the proof of Lemma 4.2.

Case $1 a$ and $b$.

$$
G_{0}=\left(\begin{array}{ccc}
1 & 0 & 0 \\
0 & 1 & 0 \\
0 & 0 & -1
\end{array}\right) \quad \text { and } \quad G_{1}=\left(\begin{array}{ccc}
a^{2} & 0 & 0 \\
0 & \pm b^{2} & 0 \\
0 & 0 & -1
\end{array}\right), \quad a, b>0
$$

Then, $\left(G_{0}^{-1} G_{1}\right)^{n}=\operatorname{diag}\left(a^{2 n},\left( \pm b^{2}\right)^{n}, 1\right)$. This is the identity matrix only for $a=$ $b=1$ which is excluded. Thus, if $G_{0}, G_{1}$ have four intersections, they cannot be part of a closed chain of conjugate conics.

Cases 2. By Theorem 3.3 we may assume that

$$
G_{0}=\left(\begin{array}{ccc}
1 & 0 & 0 \\
0 & 1 & 0 \\
0 & 0 & -1
\end{array}\right) \quad \text { and } \quad G_{1}=\left(\begin{array}{ccc}
1 & 0 & -a \\
0 & 1 & 0 \\
-a & 0 & a^{2}-r^{2}
\end{array}\right)
$$

for some $0 \leq a$ and $r>0$. So, $G_{0}$ is the unit circle and $G_{1}$ is a circle with center $(a, 0,1)$ and radius $r$. Observe, that for any $k$ the second element of the diagonal of $\left(G_{0}^{-1} G_{1}\right)^{k}$ equals 1 , and $\operatorname{det}\left(G_{0}^{-1} G_{1}\right)=r^{2}$. Hence, it follows from $\left(G_{0}^{-1} G_{1}\right)^{n}=I$, that $r=1$ and hence $a>2$. But then, $G_{i+1}$ is nested inside $G_{i}$ for each $i>1$ and the chain cannot be closed.

Case 3. As in Case 2, we may assume

$$
G_{0}=\left(\begin{array}{ccc}
1 & 0 & 0 \\
0 & 1 & 0 \\
0 & 0 & -1
\end{array}\right) \quad \text { and } \quad G_{1}=\left(\begin{array}{ccc}
1 & 0 & -a \\
0 & 1 & 0 \\
-a & 0 & a^{2}-r^{2}
\end{array}\right), \quad a \geq 0
$$


and as above, we conclude that $r=1$, and therefore $a \neq 0$. Hence,

$$
G_{1}=\left(\begin{array}{ccc}
1 & 0 & -a \\
0 & 1 & 0 \\
-a & 0 & a^{2}-1
\end{array}\right)
$$

Next, we move the two unit circles $\frac{a}{2}$ to the left and exchange the axes $x_{2}$ and $x_{3}$. Then, since we assume that the two conics intersect in two points, we have $a<2$ and by a suitable scaling of the $x_{1}$ and $x_{2}$ axes, we arrive at the following form:

$$
\tilde{G}_{0}=\left(\begin{array}{ccc}
\frac{\sqrt{4-a^{2}}}{2} & \frac{a}{2} & 0 \\
\frac{a}{2} & -\frac{\sqrt{4-a^{2}}}{2} & 0 \\
0 & 0 & 1
\end{array}\right) \quad \text { and } \quad \tilde{G}_{1}=\left(\begin{array}{ccc}
\frac{\sqrt{4-a^{2}}}{2} & -\frac{a}{2} & 0 \\
-\frac{a}{2} & -\frac{\sqrt{4-a^{2}}}{2} & 0 \\
0 & 0 & 1
\end{array}\right)
$$

Finally, a rotation in the first two variables leads to the final form of two hyperbolas

$$
\begin{aligned}
H_{0} & =\left(\begin{array}{ccc}
-1 & 0 & 0 \\
-0 & 1 & 0 \\
-0 & 0 & 1
\end{array}\right) \text { and } H_{\alpha}=\left(\begin{array}{ccc}
-\left(1-\frac{a^{2}}{2}\right) & \frac{a}{2} \sqrt{4-a^{2}} & 0 \\
\frac{a}{2} \sqrt{4-a^{2}} & 1-\frac{a^{2}}{2} & 0 \\
0 & 0 & 1
\end{array}\right) \\
& =\left(\begin{array}{ccc}
-\cos (\alpha) & \sin (\alpha) & 0 \\
\sin (\alpha) & \cos (\alpha) & 0 \\
0 & 0 & 1
\end{array}\right)
\end{aligned}
$$

where $0<\alpha<\pi$. Now, the condition $\left(H_{0}^{-1} H_{\alpha}\right)^{n}=I$, and $n$ minimal with this property, is equivalent to $\alpha=\frac{2 \pi}{n}$. So, this is the standard form if the two initial conics have two points of intersection.

Case 4. Here,

$$
G_{0}=\left(\begin{array}{ccc}
1 & 0 & 0 \\
0 & 1 & 0 \\
0 & 0 & -1
\end{array}\right) \quad \text { and } \quad G_{1}=\left(\begin{array}{ccc}
\mu+1 & 0 & -\mu \\
0 & 1-\mu & 0 \\
-\mu & 0 & \mu-1
\end{array}\right), \quad \mu \notin\{0,1\}
$$

Then, for each $n,\left(G_{0}^{-1} G_{1}\right)^{n}=: H_{n} \neq I$, since the third element of the first column of $H_{n}$ equals $n \mu$.

Case 5. By the same reasoning as in Case 2 above, we may exclude that a closed chain of conjugate conics exist such that two consecutive conics have one first order contact.

Case 6. Here,

$$
G_{0}=\left(\begin{array}{ccc}
1 & 0 & 0 \\
0 & 1 & 0 \\
0 & 0 & -1
\end{array}\right) \text { and } G_{1}=\left(\begin{array}{ccc}
1 & 0 & 0 \\
0 & 1-\mu & 0 \\
0 & 0 & -1
\end{array}\right), \mu \neq 0
$$


Then, $\left(G_{0}^{-1} G_{1}\right)^{n}=\operatorname{diag}\left(1,(1-\mu)^{n}, 1\right)=I$ iff $(1-\mu)^{n}=1$. Then either $\mu=0$ (which is excluded) or $\mu=2$ and $n=2$ (recall that $n$ is supposed to be minimal). By exchanging the $x_{2}$ - and the $x_{3}$-axis, we find

$$
G_{0}=\left(\begin{array}{ccc}
1 & 0 & 0 \\
0 & -1 & 0 \\
0 & 0 & 1
\end{array}\right) \text { and } G_{1}=\left(\begin{array}{ccc}
1 & 0 & 0 \\
0 & -1 & 0 \\
0 & 0 & -1
\end{array}\right)
$$

as standard form of the only existing closed chain of conjugate conics such that two consecutive conics have two first order contacts. Such a chain has necessarily length 2.

Case 7. Here,

$$
G_{0}=\left(\begin{array}{ccc}
1 & 0 & 0 \\
0 & 1 & 0 \\
0 & 0 & -1
\end{array}\right) \quad \text { and } G_{1}=\left(\begin{array}{ccc}
1 & -\mu & 0 \\
-\mu & 1 & \mu \\
0 & \mu & -1
\end{array}\right), \quad \mu \neq 0
$$

Then, the second element of the first row of $\left(G_{0}^{-1} G_{1}\right)^{n}$ equals $-n v \neq 0$. Hence in this situation, no closed chain of conjugate conics exists.

Case 8. Here,

$$
G_{0}=\left(\begin{array}{ccc}
1 & 0 & 0 \\
0 & 1 & 0 \\
0 & 0 & -1
\end{array}\right) \quad \text { and } \quad G_{1}=\left(\begin{array}{ccc}
1 & 0 & 0 \\
0 & \mu+1 & -\mu \\
0 & -\mu & \mu-1
\end{array}\right), \quad \mu \neq 0
$$

Then, the third element of the second row of $\left(G_{0}^{-1} G_{1}\right)^{n}$ equals $-n v \neq 0$. Hence in this situation, no closed chain of conjugate conics exists either.

\subsection{Miraculous chains of Poncelet triangles and quadrilaterals}

By geometrical arguments, it is easy to see that the closed chain of conjugate conics of length 2 in Case 6 in the previous section cannot carry Poncelet polygons. Hence, the only chance to find closed chains of conjugate conics which carry Poncelet polygons are $H_{0}$ and $H_{1}$ from Case 3 in the previous section.

For $D(\lambda)=\operatorname{det}\left(H_{0}+\lambda H_{1}\right)$ and $\sqrt{D(\lambda)}=c_{0}+c_{1} \lambda+c_{2} \lambda^{2}+c_{3} \lambda^{3}+\ldots$ we compute

$$
\begin{aligned}
c_{0}=1, \quad c_{1} & =\frac{1}{2}+\cos (\alpha), \quad c_{2}=-\frac{1}{8}+\frac{\cos (\alpha)}{2}+\frac{\sin (\alpha)^{2}}{2} \\
c_{3} & =\frac{1}{16}-\frac{\cos (\alpha)}{8}+\frac{\sin (\alpha)^{2}}{4}-\frac{\cos (\alpha) \sin (\alpha)^{2}}{2}, \ldots
\end{aligned}
$$


In order to get a miraculous chain of Poncelet triangles, we must find $0<\alpha<\pi$ such that $\alpha=\frac{2 \pi}{n}$ (for some $n \geq 3$ ) and $c_{2}=0$. If we replace $\cos (\alpha)$ with $t$, then the equation $c_{2}=0$ is equivalent to the equation

$$
(-3+2 t)(1+2 t)=0 .
$$

As solutions we get $t_{1}=\frac{3}{2}$ and $t_{2}=-\frac{1}{2}$. Since $-1 \leq \cos (\alpha) \leq 1$, there is no $0<\alpha \pi$ such that $t_{1}=\cos (\alpha)$. On the other hand, for $\alpha=\frac{2 \pi}{3}$ we get $t_{2}=\cos (\alpha)$. Hence, up to projective transformations, there is exactly one miraculous chain of Poncelet triangles. The figure below shows the canonical miraculous chain with three hyperbolas and three triangles.

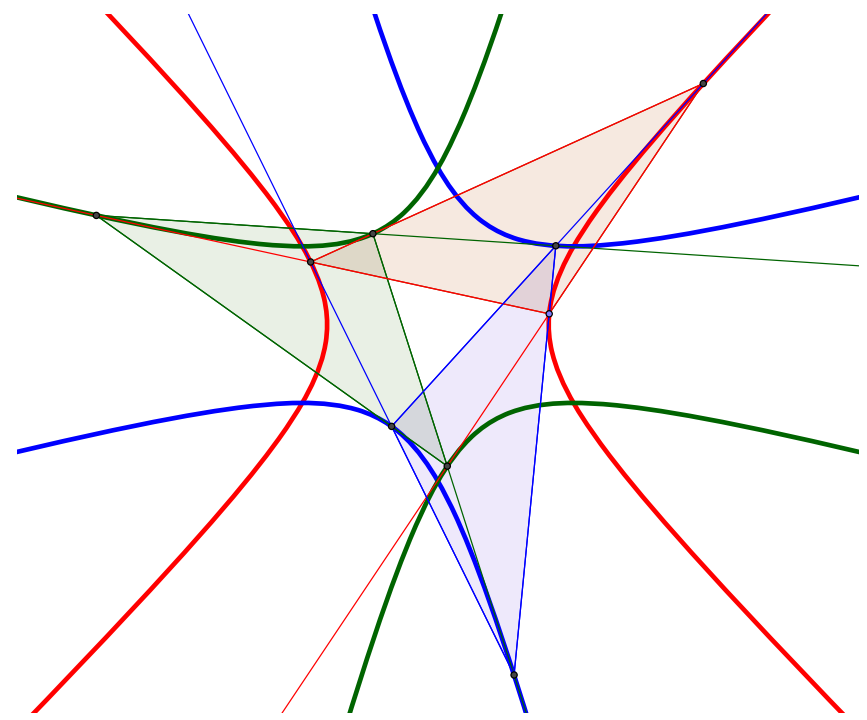

Canonical miraculous chain of Poncelet triangles

In order to get a miraculous chain of Poncelet quadrilaterals, we must find $0<\alpha<$ $\pi$ such that $\alpha=\frac{2 \pi}{n}$ (for some $n \geq 3$ ) and $c_{3}=0$. If we replace $\cos (\alpha)$ again with $t$, then the equation $c_{3}=0$ is equivalent to the equation

$$
(-1+2 t)\left(-5+4 t^{2}\right)=0 \text {. }
$$

The only solution with $-1<t<1$ is $t=\frac{1}{2}$, which gives us $\alpha=\frac{\pi}{3}$. Hence, up to projective transformations, there is exactly one miraculous chain of Poncelet quadrilaterals. The figure below shows the canonical miraculous chain with six hyperbolas and two of the six quadrilaterals. 


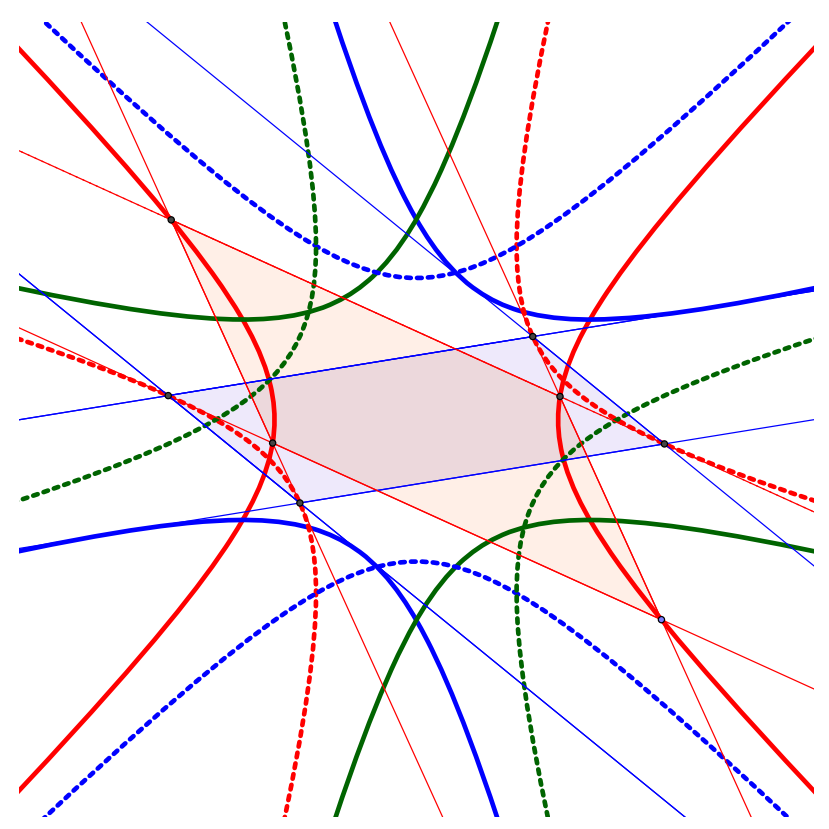

Canonical miraculous chain of Poncelet quadrilaterals

\subsection{Are there other miraculous chains of Poncelet polygons?}

In order to get a miraculous chain of Poncelet pentagons, we must find $0<\alpha<\pi$ such that $\alpha=\frac{2 \pi}{n}$ (for some $n \geq 3$ ) and $c_{2} c_{4}-c_{3}^{2}=0$. For $t=\cos (\alpha)$, the latter equation is equivalent to the equation

$$
\left(17-10 t-20 t^{2}+8 t^{3}\right)\left(-11+6 t-4 t^{2}+8 t^{3}\right)=0
$$

where the two factors are irreducible. Now, if $\cos (\alpha)$ is a root of one of these factors, where $\alpha=\frac{2 \pi}{n}$ (for some $n \geq 3$ ), then, since cyclotomic polynomials are irreducible, all roots of that factor must be in the open interval $(-1,1)$. With Sturm's Theorem one can now count, how many roots of the factors $17-10 t-20 t^{2}+8 t^{3}$ and $-11+6 t-4 t^{2}+8 t^{3}$ respectively, belong to $[-1,1]$. Since this number is less than 3 (which is the degree of the factors), we can be sure that the factor does not have a root of the form $\cos \left(\frac{2 \pi}{n}\right)$, i.e., there is no miraculous chain of Poncelet pentagons.

In order to show that there is no miraculous chain of Poncelet hexagons, we consider the equation $c_{3} c_{5}-c_{4}^{2}=0$, which is equivalent to the equation

$$
(-3+2 t)(1+2 t)\left(-7+4 t+4 t^{2}\right)\left(-41+64 t-8 t^{2}-64 t^{3}+48 t^{4}\right)=0 .
$$

Since the root of the factor $1+2 t$ leads to $\alpha=\frac{2 \pi}{3}$, the hexagon is just a triangle run through twice. Thus, we have just to consider the other three factors and with the same arguments as above we can show that there are no miraculous chains of Poncelet hexagons. 
With this technique, we have shown that except for $k=3$ and $k=4$, there are no miraculous chains of Poncelet $k$-gons for $k \leq 23$. This motivates the following

Conjecture There are no miraculous chains of Poncelet $k$-gons for $k \geq 5$.

Open Access This article is distributed under the terms of the Creative Commons Attribution 4.0 International License (http://creativecommons.org/licenses/by/4.0/), which permits unrestricted use, distribution, and reproduction in any medium, provided you give appropriate credit to the original author(s) and the source, provide a link to the Creative Commons license, and indicate if changes were made.

\section{References}

Bix, R.: Conics and Cubics: A Concrete Introduction to Algebraic Curves. Undergraduate Texts in Mathematics. Springer, New York (2006)

Bos, H.J.M., Kers, C., Oort, F., Raven, D.R.: Poncelet's Closure Theorem. Expos. Math. 5(4), 289-364 (1987)

Bryant, R.L.: Poncelet's Theorem (2010). http://mathcircle.berkeley.edu/archivedocs/2010_2011/lectures/ 1011lecturespdf/PonceletforBMC.pdf. Accessed 11 Jan 2017

Cayley, A.: Developments on the porism of the in-and-circumscribed polygon. Philos. Mag. 7, 339-345 (1854)

Chapple, W.: An essay on the properties of triangles inscribed in and circumscribed about two given circles. Misc. Curiosa Math. 1(4), 117-124 (1749)

Cieślak, W., Martini, H., Mozgawa, W.: On the rotation index of bar billiards and Poncelet's porism. Bull. Bel. Math. Soc. Simon Stev. 20(2), 287-300 (2013)

Cieślak, W., Mozgawa, W.: In search of a measure in Poncelet's porism. Acta Math. Hungar. 149(2), 338-345 (2016)

Del Centina, A.: oncelet's porism: a long story of renewed discoveries. I. Arch. Hist. Exact Sci. 70(1), 1-122 (2016)

Del Centina, A.: Poncelet's porism: a long story of renewed discoveries. II. Arch. Hist. Exact Sci. 70(2), 123-173 (2016)

Dragović, V., Radnović, M.: Poncelet Porisms and Beyond: Integrable Billiards, Hyperelliptic Jacobians and Pencils of Quadrics. Frontiers in Mathematics. Springer, New York (2011)

Euler, L.: Solutio facilis problematum quorundam geometricorum difficillimorum. Novi Commentarii academiae scientiarum imperialis Petropolitanae 11, 103-123 (1767)

Flatto, L.: Poncelet's Theorem. American Mathematical Society, Providence (2009)

Fuss, N.: De polygonis symmetrice irregularibus circulo simul inscriptis et circumscriptis. Nova Acta academiae scientiarum imperialis petropolitanae. 13, 166-189 (1795)

Halbeisen, L., Hungerbühler, N.: A simple proof of Poncelet's theorem (on the occasion of its bicentennial). Am. Math. Monthly 122, 537-551 (2015)

Halbeisen, L., Hungerbühler, N.: Conjugate conics and closed chains of Poncelet polygons. Mitteilungen der Mathematischen Gesellschaft in Hamburg 36, 5-28 (2016)

Hong, Y.P., Horn, R.A., Johnson, C.R.: On the reduction of pairs of Hermitian or symmetric matrices to diagonal form by congruence. Linear Algebra Appl. 73, 213-226 (1986)

King, J.L.: Three problems in search of a measure. Am. Math. Monthly 101, 609-628 (1994)

Lord, E.: Symmetry and Pattern in Projective Geometry. Springer, London (2012)

Par un Abonné: Questions résolues. Ébauche de solution du problème de géométrie proposé à la page 128 de ce recueil. Ann. Math. Pures Appl. 7, 373-379 (1816-1817)

Pesonen, E.: Über die Spektraldarstellung quadratischer Formen in linearen Räumen mit indefiniter Metrik. Ann. Acad. Sci. Fenn. Ser. A. I. 227, 31 (1956)

Petitjean, S.: Invariant-based characterization of the relative position of two projective conics. Nonlinear Computational Geometry, IMA Vol. Math. Appl. vol. 151, pp. 189-220, Springer, New York (2010)

Poncelet, J.-V.: Traité des propriétés projectives des figures. Bachelier, Paris (1822)

Questions proposées: Problème de géométrie. Ann. Math. Pures Appl. 7, 128 (1816-1817)

Steiner, J.: Geometrische Aufgaben und Lehrsätze. J. Regine Angew. Math. 2, 96-98 (1827a)

Steiner, J.: Geometrische Lehrsätze. J. Regine. Angew. Math. 2, 287-292 (1827b)

Vallès, J.: A vector bundle proof of Poncelet's closure theorem. Exp. Math. 30(4), 399-405 (2012) 